\title{
Opioid use, motivation to quit, and treatment status related to COVID-19: a cross-sectional study
}

\author{
Maria A. Parker ${ }^{1^{*}}$ (D) Jon Agley ${ }^{2}$, Zachary W. Adams ${ }^{3}$ and Andrea C. Villanti ${ }^{4}$
}

\begin{abstract}
Objective: Persons who use opioids may be at elevated risk of harm from the coronavirus disease 2019 (COVID-19) pandemic, yet few data currently exist that can be used to examine this risk. As part of a rapid response survey, this study measured opioid users' perceptions of risk or harm from COVID-19, as well as potential changes in motivation to quit, frequency of use, and engagement with treatment. Data collected from Amazon's Mechanical Turk ( $n=562)$ were analyzed.

Results: Participants perceived modest risk elevation from COVID-19 due to their opioid use, and perceived moderate risk to themselves or their community from COVID-19. Since learning about COVID-19, 31.2\% reported decreasing their opioid use, and $26.0 \%$ reported increased motivation to quit. Thirty-seven percent of participants reported both their use and motivation to quit stayed the same; $16.6 \%$ reported decreased use and increased motivation to quit. Participants who reported that their opioid use increased after learning about COVID-19, or whose motivation to quit opioids decreased, were more likely to also be engaged in treatment than those whose use or motivation stayed the same. These preliminary findings suggest that there likely is an association between COVID-19, opioid use, and treatment engagement that merits further in-depth investigation.
\end{abstract}

Keywords: Opioids, Survey, Motivation, COVID-19, Treatment

\section{Introduction}

Major disasters long have been associated with substantive adverse outcomes, including anxiety and substance use [1]. Similarly, the coronavirus 2019 (COVID-19; Severe Acute Respiratory Syndrome Coronavirus 2 [SARS-CoV-2]) pandemic is likely to pose serious problems for persons with opioid use disorder (OUD) [2]. Although the health consequences of the pandemic remain unclear [3], evidence suggests there have been challenges in maintaining substance use disorder treatment services [4], and a recent content review of

\footnotetext{
*Correspondence: map2@iu.edu

1 Department of Epidemiology \& Biostatistics, Indiana University School of Public Health, Bloomington, IN, USA

Full list of author information is available at the end of the article
}

COVID-19 and addiction suggests risk of increases in prevalence of withdrawal symptoms and addictive behaviors, including relapse [5].

Persons with substance use disorders, such as OUD, are likely at greater risk of worse COVID-19 outcomes $[6,7]$. For example, individuals who use opioids may be more likely than others to experience worsened hypoxemia or be subjected to "triage bias" [8]. Further, individuals with OUD have more co-occurring disorders, an increased prevalence of cigarette smoking [9], and often live in situations where social distancing is not possible [10]. A growing body of literature has also focused on the "collision" of the COVID-19 pandemic and opioid epidemic $[10,11]$, with attention to persons with psychiatric comorbidities. Finally, there is preliminary evidence of an 
increase in opioid overdoses during the early months of the pandemic, compared to a pre-COVID period $[12,13]$.

Amidst the ongoing pandemic, data remain sparse [3], and there has been little research to assess whether COVID-19 has affected opioid use and motivation to stop using opioids. Preliminary studies show some increases in opioid use through drug testing data $[14,15]$. However, the issue is complex and will require concerted effort from the field to address. To facilitate rapid response and data collection, the authors were able to collect data as part of a multiple-team, combined questionnaire administration for multiple types of substance use. The goal of this specific component of the study was to explore the relationship between treatment for OUD, changes in opioid use, and changes in motivation to stop using opioids among persons who use opioids, in the context of the COVID-19 pandemic.

\section{Main text}

\section{Materials and methods}

\section{Data collection}

An online survey using web-based crowdsourcing was conducted via Amazon Mechanical Turk (mTurk) between April 22 and May 17, 2020. The overall survey was designed to examine the relationship between treatment for OUD and cigarette smoking among persons with recent opioid use [17]. For this research note, questions related to COVID-19 were included because of the importance of assessing the impact of the pandemic on persons who use opioids.

Screened participants $(n=1022)$ were ages 18 and older, were United States residents, had used opioids in their lifetime, and had a Human Intelligence Task (HIT) approval rate greater than 90 . 'HIT' is the name for the virtual tasks (i.e., question that needs an answer) posted on mTurk that potential respondents can complete and submit in exchange for payment of a predetermined amount of money if the task is completed as intended (i.e., "approved") (see www.mturk.com for more detail). The analytic subsample for this study comprised persons who endorsed any past-month prescription opioid misuse or heroin use $(n=600)$ and answered all COVID-related survey questions $(\mathrm{n}=562$ [94\% response rate]) (see "Measures"). While "crowdsourced" data collection using mTurk is relatively new, evidence suggests that it compares favorably to other types of participant pools $[18,19]$, tends to be reliable and valid [20], and in many ways reflects the overall US population [21], though there are also some differences (e.g., intellectual abilities and level of education) [22]. Using a competing platform that is similar to mTurk, researchers were also able to replicate well-established experimental findings [23]. For this study, in response to recent concerns about non-compliant mTurk workers [24], checks were embedded in the Qualtrics survey to eliminate potential bots, avoid duplicate responses, and ensure eligibility.

\section{Measures}

Sociodemographics Respondents were asked to indicate their sex [male/female], age, race/ethnicity [White, Black, Hispanic, Other], and education $[\leq$ High School, Some College, Bachelor's, $>4$ years College].

Opioid use and treatment Participants were asked, "Have you ever, even once, used any prescription pain reliever in any way a doctor did not direct you to use it, including using it without a prescription of your own, using it in greater amounts, more often, or longer than you were told to take it, or using it in any other way a doctor did not direct you to use it?" [25, 26]. This was followed by questions that asked how many days in the last month respondents used (1) prescription opioids or (2) heroin. An additional question asked, "Are you currently enrolled in a treatment program for your prescription opioid or heroin use?".

COVID-19 perceptions, opioid use and motivation to quit Items on COVID-19 were adapted from a recent tobacco study [16]. Participants were asked, "How concerned about coronavirus (COVID-19) are you for your own health?" and "How concerned about coronavirus (COVID-19) are you for the health of others in your community?" both with response options ranging from $[0=$ Not at all to $7=$ Extremely $]$. Then, participants were asked, "Do you think your opioid use increases your risk of harm from coronavirus (COVID-19)?" [0= Definitely no to $7=$ Definitely yes] [16]. Finally, respondents answered questions about opioid use and motivation to quit: "How has your opioid use changed since you learned about the coronavirus pandemic (COVID-19)?", "How has your motivation to quit opioid use changed since you learned about the coronavirus pandemic (COVID-19)?" Each question allowed participants to select a nominal response option from: decreased, increased, stayed the same, other (please specify) [16].

Analytic plan Prevalence values and means were calculated for background characteristics and COVID-19 related questions. Next, cross-tabulations between opioid use and motivation to quit were completed, followed by a logistic regression analysis to estimate the association between treatment as the outcome (yes/no) and opioid use (decreased/increased/stayed the same) and motivation to quit (decreased/increased/stayed the same) as the predictors of interest. An adjusted logistic regression model followed, which controlled for covariates (sex, age, race/eth- 
nicity, education, and perception of whether opioid use increased risk of harm during COVID-19). In sensitivity analyses, for the 'other' category of opioid use and motivation to quit, 57 responses were persons who reported not using opioids since learning about COVID-19 (although within the past month). Therefore, the adjusted analyses were conducted after removing persons who specified they no longer used opioids amid the pandemic $(n=505)$. In other words, missing data was addressed with complete case analysis (listwise deletion). The model was checked for multicollinearity using the 'collin' command. All analyses were completed in Stata, version 16.

\section{Results}

Descriptive outcomes: sociodemographics, opioid use and treatment, COVID-19 perceptions, opioid use and motivation to quit

The sample was $51 \%$ female, $67 \%$ Non-Hispanic White, $8 \%$ Non-Hispanic Black, and 15\% Hispanic, with a median age of 34. Participants reported moderate concern about COVID-19 for their own health (mean 4.8; $\mathrm{SD}=1.8)$, and for the health of others in their community (mean $5.3 ; \mathrm{SD}=1.6$ ); they were ambivalent about the idea that their opioid use increased their risk of harm from COVID-19 (mean $=4.1, \mathrm{SD}=2.0$ ). Approximately $22.1 \%(n=124)$ of participants were actively enrolled in a treatment program for their opioid use. Similar proportions reported that their opioid use (46.6\%) and motivation to quit opioid use (54.4\%) stayed the same since learning about the coronavirus pandemic, whereas $31.3 \%$ of participants' use decreased, and $26.0 \%$ of participants' motivation to quit increased (Fig. 1). In examining the correlation between use and motivation, 37.5\% of participants' use and motivation stayed the same and $16.6 \%$ of individuals reported both that they decreased their use and their motivation to quit increased.
Regression outcomes: association between treatment, opioid use, and motivation to quit

In adjusted analyses, participants whose opioid use increased (AOR $=3.8 ; 95 \% \mathrm{CI} 2.0,7.5)$ and whose motivation to quit opioids decreased $(\mathrm{AOR}=3.3 ; 95 \% \mathrm{CI}$ $1.7,6.3)$ were significantly more likely to be in treatment than persons whose use or motivation stayed the same, respectively. Persons who decreased their opioid use were significantly less likely to be in treatment than those whose use stayed the same (AOR $=0.5 ; 95 \%$ CI 0.2, 0.9; Table 1).

\section{Discussion}

Descriptive outcomes: sociodemographics, opioid use and treatment, COVID-19 perceptions, opioid use and motivation to quit

Participants in this study reported relatively moderate concerns about COVID-19 for their own health. While parallel research is limited, this conceptually mirrors other findings where such concerns tend to slightly exceed the scale midpoint, such as from a US college student validation of the Fear of COVID-19 scale [27], and data collected on perceived vulnerability to and severity of COVID-19 [28]. However, these perceptions are also subject to change given the rapidly-expanding knowledge base about COVID-19.

Fewer than one-quarter (22.1\%) of participants reported being enrolled in a treatment program for prescription opioid or heroin use. Estimates of treatment utilization often vary depending on the way in which treatment is defined (e.g., outpatient office-based treatment, methadone, jail-based treatment, or group programs, among others). This study remained agnostic as to the type of treatment, except specifying the substances of focus. Analysis of data from a similar question among individuals ages 12 and older, administered via the 2005 to 2013 National Surveys on Drug Use and Health (NSDUH), identified a 19\% prevalence of opioid-specific

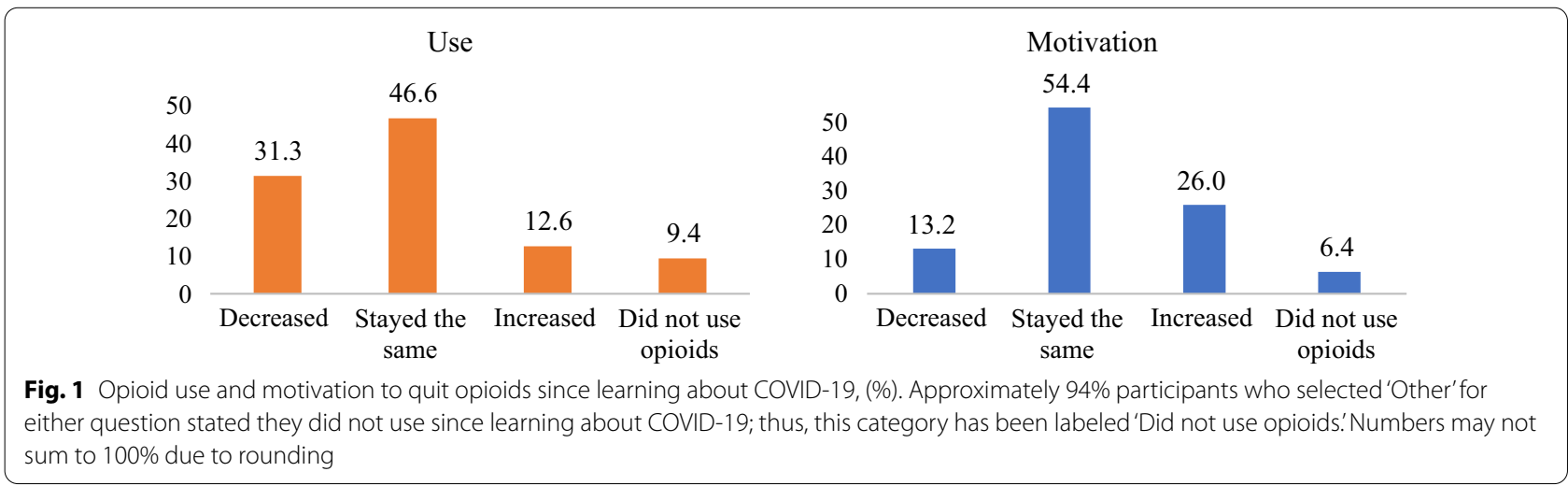


Table 1 Estimates from logistic regression with treatment as the outcome and opioid use and motivation to quit since learning about COVD-19 as the predictors of interest $(n=505)$

\begin{tabular}{|c|c|c|c|c|c|c|}
\hline \multirow{2}{*}{ Opioid use } & \multirow[t]{2}{*}{ Odds ratio } & \multicolumn{2}{|c|}{ 95\% confidence interval } & \multirow[t]{2}{*}{ AOR } & \multicolumn{2}{|c|}{$\begin{array}{l}95 \% \text { confidence } \\
\text { interval }\end{array}$} \\
\hline & & & & & & \\
\hline Decreased & 0.54 & 0.30 & 0.97 & 0.46 & 0.24 & 0.89 \\
\hline Increased & 5.15 & 2.85 & 9.30 & 3.83 & 1.96 & 7.47 \\
\hline Stayed the same & 1.00 & & & 1.00 & & \\
\hline \multicolumn{7}{|l|}{ Motivation to quit } \\
\hline Decreased & 3.82 & 2.03 & 7.17 & 3.25 & 1.67 & 6.31 \\
\hline Increased & 2.24 & 1.26 & 3.98 & 1.90 & 0.99 & 3.66 \\
\hline Stayed the same & 1.00 & & & 1.00 & & \\
\hline Male & - & & & 0.51 & 0.30 & 0.85 \\
\hline Age & - & & & 1.01 & 0.99 & 1.04 \\
\hline \multicolumn{7}{|l|}{ Race/ethnicity } \\
\hline Non-Hispanic White & - & & & 1.00 & & \\
\hline Non-Hispanic Black & - & & & 0.50 & 0.15 & 1.66 \\
\hline Hispanic & - & & & 3.47 & 1.96 & 6.15 \\
\hline Non-Hispanic Other & - & & & 0.96 & 0.38 & 2.40 \\
\hline \multicolumn{7}{|l|}{ Education } \\
\hline Bachelor's degree & - & & & 1.00 & & \\
\hline High school or less & - & & & 1.21 & 0.61 & 2.40 \\
\hline Some college & - & & & 0.35 & 0.17 & 0.72 \\
\hline Master's degree or higher & - & & & 1.13 & 0.57 & 2.25 \\
\hline $\begin{array}{l}\text { Perception that opioid use increases } \\
\text { harm from COVID-19 }\end{array}$ & - & & & 1.37 & 1.18 & 1.59 \\
\hline
\end{tabular}

Adjusted model included sex, age, race/ethnicity, education, and opioid use harm perception. These estimates reflect analyses conducted after removing persons who reported no longer using opioids since learning about COVID-19 $(n=57)$

treatment among those with OUD [29]. However, the 2015 NSDUH identified marked differences between the percentage needing vs receiving specialty treatment for illicit drug use among ages 18 and older (19.6\%) and 26 and older (24.1\%) [30]. Given the median age of this sample (36 years) and the restriction to ages $18+$, the prevalence of reported treatment engagement appears plausible, lending conceptual validity to the study.

Interestingly, the majority of individuals reported that their opioid use (46.6\%) and/or motivation to quit (54.4\%) remained the same since learning about COVID-19. Smaller percentages indicated that their use decreased (31.3\%) and/or motivation to quit increased (26.0\%) since learning about COVID-19. This stands in contrast to individuals' responses about their tobacco use, where there was an increase in tobacco product use after learning about COVID-19 [16]. Although the survey results are cross-sectional and limited by the need to collect data rapidly in response to an evolving pandemic, researchers should conduct further, rigorous investigation into the degree to which the COVID-19 pandemic may represent not just a risk to persons who use opioids, but also a potential treatment leverage point where individuals may have heightened motivation to engage with provider systems.

Finally, roughly twice as many participants reported decreased (vs. increased) opioid use and increased (vs. decreased) motivation to quit, suggesting this may be an opportune time for clinical outreach and intervention among similar individuals. The subset of our sample that reported greater motivation to stop their opioid use and/ or decreased opioid use after learning about COVID-19 represents an opportunity for innovative and 'low threshold' paths to treatment [10].

\section{Regression outcomes: association between treatment, opioid use, and motivation to quit}

The finding that increased opioid use was associated with reporting engagement with treatment, as was decreased motivation to quit, requires interpretation. Perception of harm from COVID-19 was only modestly associated with treatment engagement. An appropriate cross-sectional interpretation is that "participants who reported being in treatment were more likely to also be participants who reported increased use since learning about COVID-19." These data were not collected temporally, so engagement 
with treatment may have been a consequence, rather than an antecedent, of increased use-or the converse. Decreased motivation (relative to motivation that stayed the same) for those engaged in treatment may reflect a broader, but unmeasured, multivariate relationship between treatment, substance use, and the pandemic.

While policy changes have been implemented to improve substance use treatment during COVID, access to treatment has remained limited [4]. US Drug Enforcement Agency (DEA) guidance allows exceptions during the pandemic-related emergency such as prescribing in states where providers are not registered with the DEA using telemedicine [31], but these options require individual provider adoption. The need for mutual aid gatherings (e.g., Narcotics Anonymous) to move online was identified early [32], but barriers to access and identification remained. These findings may also reflect more complex clinical phenotypes, the role of social isolation in opioid use and overdose [7], or associations between COVID-19 and poor mental health [33]. Decreased motivation to quit may likewise have resulted from changes to treatment procedures due to COVID-19 (e.g., shift to virtual visits), but this hypothesis would need to be tested through a separate study.

\section{Conclusion}

The takeaway implication, as with the descriptive outcomes, is that there appears to be a shift in both risk and treatment access during the COVID-19 pandemic. At the same time, these data do not support the idea that this shift is driven directly by worry about COVID19 itself. Rather, it is likely associated with a more complex interplay of causal factors. This brief data collection effort provides only a surface-level examination of issues, but these data indicate the need for further and deeper examination.

\section{Limitations}

These cross-sectional data were collected as a rapidresponse addendum to an extant study [17], so they provide only an indication of the areas on which researchers might profitably focus; the findings should not be generalized or used to influence policy or clinical practice without further study. In addition, this study analyzed several conceptually related but unidimensional constructs, while the impact of the pandemic likely affected numerous additional variables directly and indirectly, such as social isolation and alteration of substance access. Results should be understood as exploratory in light of this limitation. Conducting this study online via mTurk excluded adults who do not use the Internet, though this percentage was low as of 2019 (10\%), with non-users primarily being ages 65 and older [34]. Further, key areas of difference between mTurk and the US population (age and education level) were controlled as covariates in the adjusted regression model [20-22].

\section{Abbreviations}

AOR: Adjusted odds ratio; COVID-19: Coronavirus disease 2019; DEA: US Drug Enforcement Administration; HIT: Human Intelligence Task; mTurk: Amazon Mechanical Turk; NSDUH: National Survey on Drug Use and Health; OUD: Opioid use disorder.

\section{Acknowledgements}

The authors would like to thank Justin Strickland, PhD for helping prepare the survey for Amazon Mechanical Turk. They would also like to thank the Indiana University Biostatistics Consulting Center, namely Jodi Byers, MS, Stephanie Dickinson, MS, and Beate Henschel, MPH for their expertise designing the survey and preparing the dataset for analyses.

\section{Authors' contributions}

MAP and ACV designed the study. MAP collected the data and analyzed the data. Both MAP and JDA were major contributors in writing the manuscript. ACV and ZWA provided substantiative feedback for the manuscript drafts. All authors read and approved the final manuscript.

\section{Funding}

This research was funded by the School of Public Health, Indiana UniversityBloomington [Research Start-up Funds; MAP]. ACV was supported in part by the National Institute of General Medical Sciences (NIGMS) of the NIH (P20GM103644) and by Health Resources and Services Administration (HRSA) center Grant (UD9RH33633) from the U.S. Department of Health and Human Services. The content is solely the responsibility of the authors and does not necessarily represent the official views of the $\mathrm{NIH}, \mathrm{HRSA}$, or the US Government.

\section{Availability of data and materials}

The datasets analyzed during the current study are available from the corresponding author on reasonable request.

\section{Declarations}

Ethics approval and consent to participate

Ethical approval for this study was granted by Indiana University's IRB. All participants provided written informed consent through the online survey.

\section{Consent for publication}

Not applicable.

\section{Competing interests}

The authors report no competing interests.

\section{Author details}

${ }^{1}$ Department of Epidemiology \& Biostatistics, Indiana University School of Public Health, Bloomington, IN, USA. ${ }^{2}$ Prevention Insights, Department of Applied Health Science, Indiana University School of Public Health, Bloomington, IN, USA. ${ }^{3}$ Indiana University School of Medicine, Indianapolis, IN, USA. ${ }^{4}$ Vermont Center on Behavior \& Health, University of Vermont, Burlington, VT, USA.

Received: 19 October 2020 Accepted: 6 May 2021

Published online: 20 May 2021

\section{References}

1. Galea S, Merchant RM, Lurie N. The mental health consequences of COVID-19 and physical distancing: the need for prevention and early intervention. JAMA Intern Med. 2020;180(6):817-8.

2. Henry BF, Mandavia AD, Paschen-Wolff MM, Hunt T, Humensky JL, Wu E, et al. COVID-19, mental health, and opioid use disorder: old and new 
public health crises intertwine. Psychol Trauma Theory Res Pract Policy. 2020. https://doi.org/10.1037/tra0000660.

3. Mallet J, Dubertret C, Strat YL. Addictions in the COVID-19 era: current evidence, future perspectives a comprehensive review. Prog NeuroPsychopharmacol Biol Psychiatry. 2020. https://doi.org/10.1016/j.pnpbp. 2020.110070

4. Dunlop A, Lokuge B, Masters D, Sequeira M, Saul P, Dunlop G, et al. Challenges in maintaining treatment services for people who use drugs during the COVID-19 pandemic. Harm Reduct J. 2020;17(1):26.

5. Dubey MJ, Ghosh R, Chatterjee S, Biswas P, Chatterjee S, Dubey S. COVID-19 and addiction. Diabetes Metab Syndr Rev Clin Res Rev. 2020;14(5):817-23.

6. Holloway IW, Spaulding A, Miyashita A, Randall L, King A, Frew PM COVID-19 vulnerability among people who use drugs: recommendations for global public health programs and policies. J Int AIDS Soc. 2020. https://doi.org/10.1002/jia2.25551

7. Volkow ND. Collision of the COVID-19 and addiction epidemics. Ann Intern Med. 2020:173(1):61-2.

8. Slat S, Thomas J, Lagisetty P. Coronavirus disease 2019 and opioid use-a pandemic within an epidemic. JAMA Health Forum. 2020;1(5):e200628.

9. Parker MA, Sigmon SC, Villanti AC. Higher smoking prevalence among United States adults with co-occurring affective and durg use diagnoses. Addict Behav. 2019;99:106112.

10. Khatri UG, Perrone J. Opioid use disorder and COVID-19: crashing of the crises. J Addict Med. 2020;14(4):e6-7.

11. Becker WC, Fiellin DA. When epidemics collide: coronavirus disease 2019 (COVID-19) and the opioid crisis. Ann Intern Med. 2020:173(1):59-60.

12. OchalekTA, Cumpston KL, Wills BK, Gal TS, Moeller FG. Nonfatal opioid overdoses at an urban emergency department during the COVID-19 pandemic. J Am Med Assoc. 2020. https://doi.org/10.1001/jama.2020. 17477

13. Slavona S, Rock P, Bush HM, Quesinberry D, Walsh SL. Signal of increased opioid overdose during COVID-19 from emergency medical services data. Drug Alcohol Depend. 2020;214:108176.

14. Niles JK, Gudin J, Radcliff J, Kaufman HW. The opioid epidemic within the COVID-19 pandemic: drug testing in 2020. Popul Health Manag. 2020. https://doi.org/10.1089/pop.2020.0230.

15. Morin KA, Acharya S, Eibl JK, Marsh DC. Evidence of increased fentanyl use during the COVID-19 pandemic among opioid agonist treatment patients in Ontario, Canada. Int J Drug Policy. 2021;90:103088.

16. Klemperer EM, West JC, Peasley-Miklus C, Villanti AC. Change in tobacco and electronic cigarette use and motivation to quit in response to COVID-19. Nicotine Tob Res. 2020;22(9):1622-3.

17. Parker MA, Byers JE, Villanti AC. Effect of brief nicotine corrective messaging on nicotine beliefs in persons who use opioids. Exp Clin Psychopharmacol

18. Johnson DR, Borden LA. Participants at your fingertips: using Amazon's mechanical turk to increase student-faculty collaborative research. Teach Psychol. 2012;39(4):245-51.

19. Buhrmester M, Kwang T, Gosling SD. Amazon's mechanical turk: a new source of inexpensive, yet high-quality data? Perspect Psychol Sci. 2011;6(1):3-5

20. Chandler J, Shapiro D. Conducting clinical research using crowdsourced convenience samples. Annu Rev Clin Psychol. 2016;12:53-81.
21. Keith MG, Tay L, Harms PD. Systems perspective of Amazon mechanical turk for organizational research: review and recommendations. Front Psychol. 2017:8:1359.

22. Merz ZC, Lace JW, Einstein AM. Examining broad intellectual abilities obtained within an mTurk internet sample. Curr Psychol. 2020. https://doi. org/10.1007/s12144-020-00741-0

23. Peer E, Brandimarte L, Samat S, Acquisti A. Beyond the turk: alternative platforms for crowdsourcing behavioral research. J Exp Soc Psychol. 2017:70:153-63.

24. Kim HS, Hodgins DC. Are you for real? Maximizing participant eligibility on Amazon's Mechanical Turk. Addiction. 2020. https://doi.org/10.1111/ add.15065.

25. Parker MA, Anthony JC. Epidemiological evidence on extra-medical use of prescription pain relievers: transitions from newly incident use to dependence among 12-21 year olds in the United States using metaanalysis, 2002-13. PeerJ. 2015;3:e1340. https://doi.org/10.7717/peerj. 1340.

26. SAMHSA. NSDUH 2018 Methodological Resource Book (MRB): substance abuse and mental health services administration; 2017. https:// www.samhsa.gov/data/report/nsduh-2018-methodological-resou rce-book-mrb

27. Perz CA, Lang BA, Harrington R. Validation of the fear of COVID-19 scale in a US college sample. Int J Met Health Addict. 2020. https://doi.org/10. 1007/s11469-020-00356-3.

28. Calvillo DP, Ross BJ, Garcia RJB, Smelter TJ, Rutchick AM. Political ideology predicts perceptions of the threat of COVID-19 (and susceptibility to fake news about it). Soc Psychol Personal Sci. 2020. https://doi.org/10.1177/ 1948550620940539.

29. Wu L-T, Zhu H, Swartz MS. Treatment utilization among persons with opioid use disorder in the United States. Drug Alcohol Depend. 2016:169(1):117-27.

30. SAMHSA. Results from the 2016 National Survey on Drug Use and Health: detailed tables. SAMHSA; 2017. https://www.samhsa.gov/data/sites/ default/files/NSDUH-DetTabs-2015/NSDUH-DetTabs-2015/NSDUH-DetTa bs-2015.pdf

31. DEA. Dear registrants: US Drug Enforcement Agency; 2020. https://www. deadiversion.usdoj.gov/GDP/(DEA-DC-018)(DEA067)\%20DEA\%20state\% 20reciprocity\%20(final)(Signed).pdf.

32. Faces and Voices of Recovery. Coronavirus disease 2019 (COVID-19) and the recovery community: faces and voices of recovery; 2020. https:// mailchi.mp/facesandvoicesofrecovery/covid-19_statement.

33. Rosenberg M, Luetke M, Hensel D, Kianersi S, Herbenick D. Depression and loneliness during COVID-19 restrictions in the United States, and their associations with frequency of social and sexual connections. MedRxiv. 2020;2020.05.18.20101840. https://doi.org/10.1101/2020.05.18. 20101840.

34. Pew. Internet/broadband fact sheet: Pew Research Center; 2019. https:// www.pewresearch.org/internet/fact-sheet/internet-broadband/. Updated 12 June 2019

\section{Publisher's Note}

Springer Nature remains neutral with regard to jurisdictional claims in published maps and institutional affiliations.
Ready to submit your research? Choose BMC and benefit from:

- fast, convenient online submission

- thorough peer review by experienced researchers in your field

- rapid publication on acceptance

- support for research data, including large and complex data types

- gold Open Access which fosters wider collaboration and increased citations

- maximum visibility for your research: over 100M website views per year

At BMC, research is always in progress.

Learn more biomedcentral.com/submissions 